\title{
Correction to: Quantifying the circularity of regional industrial waste across multi-channel enterprises
}

\author{
Claudia Schilkowski ${ }^{1} \cdot$ Manish Shukla ${ }^{1} \cdot$ Sonal Choudhary ${ }^{2}$
}

(c) Springer Science+Business Media, LLC, part of Springer Nature 2020

\section{Correction to: Annals of Operations Research https://doi.org/10.1007/s10479-019-03168-4}

This erratum is published due to proofing error noticed with Table 5 which had been processed as image with the red box missing in the published article.

Table 5 has been thus adjusted and original article corrected.

The original article can be found online at https://doi.org/10.1007/s10479-019-03168-4.

Manish Shukla

manish.shukla@durham.ac.uk

1 Durham University Business School, Mill Hill Lane, Durham DH1 3LB, UK

2 Management School, University of Sheffield, Sheffield S10 1FL, UK 
Table 5 Analysis of the WIO according to site category and site type in Newcastle. Source: Analysis based on: Environment Agency (2017)

\begin{tabular}{|c|c|c|c|c|c|}
\hline & & $\sqrt{\text { Volume of waste }}$ & stream in tonnes & & \\
\hline Site Category & Site Type & Waste Received & Waste Removed & Total & Output - Input \\
\hline Composting & Composting (type unknown) & $48.327,76$ & $38.018,16$ & $86.345,92$ & \begin{tabular}{|r|}
-10.310 \\
\end{tabular} \\
\hline Composting & Composting in open windrows & $24.803,43$ & 187,18 & $24.990,61$ & $-24.616 \times>$ \\
\hline Incineration & Incineration & $12.435,96$ & $8.369,25$ & $20.805,21$ & -4.067 \\
\hline Landfill & HIC LF & & $63.116,02$ & $63.116,02$ & 63.116 \\
\hline Landfill & Inert LF & $60.708,02$ & & $60.708,02$ & $-60.708 \times$ \\
\hline Landfill & Non Haz (SNRHW) LF & $1.356,72$ & $13.005,00$ & $14.361,72$ & 11.648 \\
\hline Landfill & Non Hazardous LF & $713.172,34$ & $81.871,76$ & $795.044,10$ & -631.301 \\
\hline Mobile Plant & Mobile Plant - Landspreading & $91.532,79$ & $91.532,79$ & $183.065,58$ & 0 \\
\hline Mobile Plant & Mobile Plant - Treatment & 234,26 & 234,26 & 468,52 & 0 \\
\hline MRS & Car Breaker & $3.120,57$ & $5.118,46$ & $8.239,03$ & 1.998 \\
\hline MRS & Metal Recycling & $191.381,31$ & $167.438,51$ & $358.819,82$ & -23.943 \\
\hline MAPS & Vohiclo_donollution facility & 6.26700 & 611028 & 1217728 & 257 \\
\hline MRS & Metal Recyclcing & $1.195,00$ & 809,20 & $2.004,20$ & -386 \\
\hline Processing & fvontreltous tvietalteprocessing & & $0.014,14$ & $0.014,14$ & 0.014 \\
\hline Processing & Paper Recycling & $104.338,00$ & & $104.338,00$ & -104.338 \\
\hline Storage & Storage - incinerator & 192,67 & & 192,67 & -193 \\
\hline Storage & Storage - oils & 170,56 & 177,56 & 348,12 & 7 \\
\hline Transfer & CA Site & $101.126,28$ & $100.868,06$ & $201.994,33$ & -258 \\
\hline Transfer & Clinical Waste Transfer & $119.740,41$ & $75.673,90$ & $195.414,31$ & -44.067 \\
\hline Transfer & Haz Waste Transfer & $380.608,51$ & $400.412,54$ & $781.021,05$ & 19.804 \\
\hline Transfer & Inert Waste Transfer & $48.048,77$ & $45.236,14$ & $93.284,91$ & $-2.813 \times$ \\
\hline Transfer & Non-Haz Waste Transfer & $975.096,09$ & $827.968,97$ & $1.803 .065,06$ & -147.127 \\
\hline Transfer & Non-hazardous \& hazardous HWA Site & $20.097,01$ & $20.097,01$ & $40.194,02$ & 0 \\
\hline Transfer & Asbestos Waste Transfer Station & 234,72 & 151,34 & 386,06 & -83 \\
\hline Treatment & Biological Treatment & $33.136,55$ & $59.791,53$ & $92.928,08$ & 26.655 \\
\hline Treatment & $\mathrm{HCl}$ Waste TS + treatment & $26.860,50$ & $25.195,61$ & $52.056,11$ & -1.665 \\
\hline Treatment & $\mathrm{HCl}$ Waste TS + treatment + asbestos & $2.199,91$ & $2.818,85$ & $5.018,76$ & 619 \\
\hline Treatment & Inert \& excavation Waste TS + treatment & $242.052,45$ & $33.655,74$ & $275.708,19$ & -208.397 \\
\hline Treatment & Material Recycling Facility (MRF) & $216.034,24$ & $158.739,31$ & $374.773,56$ & -57.295 \\
\hline Treatment & Non-specified Treatment & $19.106,71$ & $16.398,00$ & $35.504,71$ & -2.709 \\
\hline Treatment & Physical Treatment & $162.107,57$ & $143.648,96$ & $305.756,53$ & -18.459 \\
\hline Treatment & Recovery of Waste & $184.888,69$ & $177.122,54$ & $362.011,23$ & -7.766 \\
\hline Treatment & Sewage sludge treatment & $161.248,02$ & $52.691,90$ & $213.939,92$ & -108.556 \\
\hline New & New Type & $2.179,00$ & $1.234,50$ & $3.413,50$ & -945 \\
\hline $\mathrm{N} / \mathrm{a}$ & & $99.339,26$ & $4.538,91$ & $103.878,17$ & -94.800 \\
\hline Total & & 4.053 .441 & 2.628 .846 & 6.682 .287 & -1.424 .595 \\
\hline
\end{tabular}

Publisher's Note Springer Nature remains neutral with regard to jurisdictional claims in published maps and institutional affiliations. 